\title{
flora robotica - Mixed Societies of Symbiotic Robot-Plant Bio-Hybrids
}

\author{
Heiko Hamann ${ }^{1}$, Mostafa Wahby ${ }^{1}$, Thomas Schmickl ${ }^{2}$, Payam Zahadat ${ }^{2}$, \\ Daniel Hofstadler ${ }^{2}$, Kasper Støy ${ }^{3}$, Sebastian Risi ${ }^{3}$, Andres Faiña ${ }^{3}$, \\ Frank Veenstra ${ }^{3}$, Serge Kernbach ${ }^{4}$, Igor Kuksin ${ }^{4}$, Olga Kernbach ${ }^{4}$, \\ Phil Ayres ${ }^{5}$, Przemyslaw Wojtaszek ${ }^{6}$ \\ ${ }^{1}$ Heinz Nixdorf Institute, Department of Computer Science, University of Paderborn, \\ Paderborn, Germany \\ (heiko.hamann@uni-paderborn.de) \\ ${ }^{2}$ Artificial Life Lab of the Department of Zoology, Karl-Franzens University Graz, \\ Graz, Austria \\ ${ }^{3}$ Robotics, Evolution and Art Lab (REAL), IT University of Copenhagen, \\ Copenhagen, Denmark \\ ${ }^{4}$ Cybertronica UG, Stuttgart, Germany \\ ${ }^{5}$ Centre for Information Technology and Architecture, Copenhagen, Denmark \\ ${ }^{6}$ Department of Molecular and Cellular Biology, Adam Mickiewicz University, \\ Poznań, Poland
}

September 14, 2015

\begin{abstract}
Besides the life-as-it-could-be driver of artificial life research there is also the concept of extending natural life by creating hybrids or mixed societies that are built from both natural and artificial components. In this paper, we motivate and present the research program of the project flora robotica. We present our concepts of control, hardware design, modeling, and human interaction along with preliminary experiments. Our objective is to develop and to investigate closely linked symbiotic relationships between robots and natural plants and to explore the potentials of a plant-robot society able to produce architectural artifacts and living spaces. These robot-plant bio-hybrids create synergies that allow for new functions of plants and robots. They also create novel design opportunities for an architecture that fuses the design and construction phase. The bio-hybrid is an example of mixed societies between 'hard artificial and "wet natural life, which enables an interaction between natural and artificial ecologies. They form an embodied, selforganizing, and distributed cognitive system which is supposed to grow and develop over long periods of time resulting in the creation of meaningful architectural structures. A key idea is to assign equal roles to robots and plants in order to create a highly integrated, symbiotic system. Besides the gain of knowledge, this project has the objective to create a bio-hybrid system with a defined function and application - growing architectural artifacts.
\end{abstract}

[to be published in Proc. of IEEE Symposium on Artificial Life (IEEE ALIFE'15), 2015] 


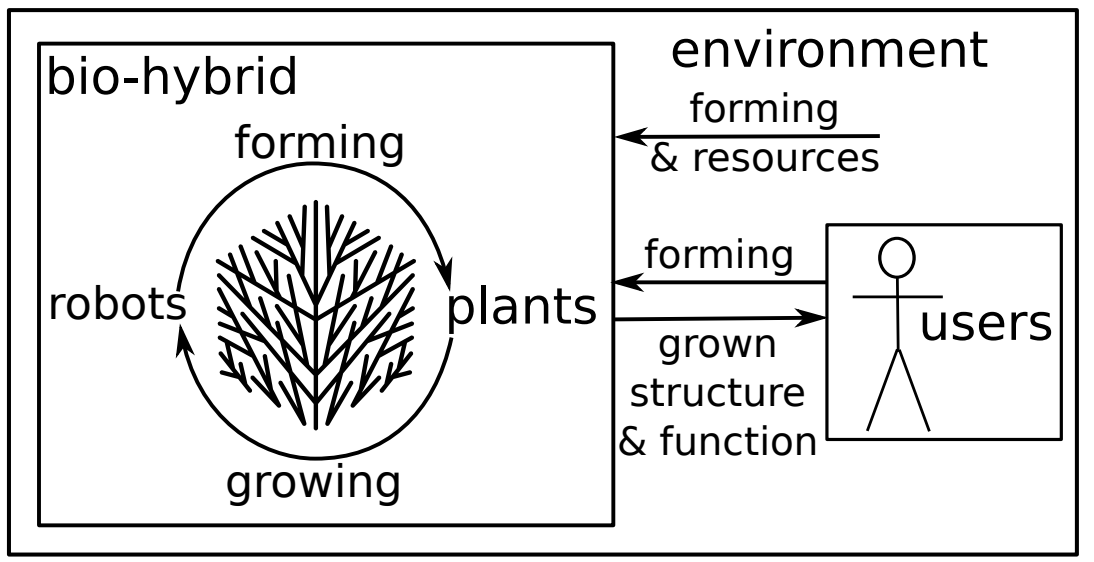

Figure 1: Schematic overview of agents and feedbacks in the bio-hybrid flora robotica system. Agents are natural plants, distributed robots, and human beings (users). Natural stimuli imposed by the environment (e.g., light, gravity, humidity) form the system but also artificial stimuli imposed by robots and users form the system. The plants provide the system with inexpensive production of material (growth). The environment provides the required resources. The bio-hybrid system provides users with a desired structure that has a desired function (architectural artifacts).

\section{Introduction}

The primary motivation of artificial life research is to study life as it could be. A secondary aspect is, for example, to study so-called 'mixed societies' of artificial life interacting with living organisms [Halloy et al., 2007, LEURRE, 2015, ASSISI $\left.\left.\right|_{b f}, 2015\right]$. Research on mixed societies is aimed at gaining a better understanding of adaptive behavior [Sempo et al., 2006], means of communication between artificial and natural life-forms [ASSISI $\left.\left.\right|_{b f}, 2015\right]$, and has also explicit applications, such as pest control, for example by controlling insect swarms such as cockroaches and locusts [Halloy et al., 2007]. Most research of mixed societies was done on mixing animals, such as cockroaches [Halloy et al., 2007], crickets [da Silva Guerra et al., 2010], bees [ASSISI $\left.\left.\right|_{b f}, 2015\right]$, and chickens [Gribovskiy et al., 2010] with robots. In this paper we present the research program of our new, interdisciplinary project 'flora robotica' which creates and investigates mixed societies of robots and natural plants (see Fig. 1 for a schematic overview) and brings together scientists from plant science, architecture, zoology, robotics, and computer science. The general idea of using robots to treat plants is obviously not novel and often has the objective of automating gardening tasks [Correll et al., 2010] or on bigger scales automating agriculture [Slaughter et al., 2008]. However, our objectives go beyond the idea of mere automation.

A project that is similar to flora robotica is PLEASED [2015], which investigates plants as bio-sensors. We also plan to use plants as sensors (phyto-sensing) but a key ingredient are robots and the formation of physical structures. A bit closer to our ideas is the concept of a distributed robot garden [Correll et al., 2009] although that is still driven by the main idea of automation. Instead, our motivation in flora robotica is not to automate gardening but to develop a bio-hybrid system that assigns equal roles to plants and robots and creates synergies between them; see the circle of feedbacks on the left-hand side of Fig. 1. Natural plants provide growth of structures, sensing capabilities, and beauty, while robots impose artificial 
stimuli in addition to natural stimuli of the environment, extended the plants' sensing and decision-making capabilities. Hence, the robots can influence the natural growth process to trigger artificial growth. On the one hand, we want to leverage natural adaptive behavior in plants [Garzón and Keijzer, 2011] in a way that extends the capabilities of robots through closely linked interactions. On the other hand, we want to leverage the free programmability of robots such that we can influence the plants in desired ways and create artificial growth processes. Hence, one of our key ideas is to extend the already rich and manifold variety of natural growth processes [Thompson, 1917] with additional, artificial growth processes of bio-hybrid systems. Such an approach has many potential applications, from which we have picked the artificial growth of architectural artifacts. The flora robotica system is therefore supposed to have an additional function besides supporting homeostasis (i.e., keep plant features close to desired levels). For example, the system could serve as a green wall that also adapts to the needs of humans who interact with it (see lower right-hand side of Fig. 1). The wall could keep holes where required and could also adapt to solar radiation and temperature (cf. 'climate-responsiveness' [Menges and Reichert, 2012]). Another function of the flora robotica system is to serve as a sensor in the form of a collective phyto-sensing system. In the following, we describe our objectives, methods, and the potential impact on the artificial life community.

\section{The bio-hybrid flora robotica system}

Instead of applications in gardening or agriculture we go for the production of architectural artifacts. The bio-hybrid flora robotica system grows into architectural structures, such as walls, roofs, and benches, providing functionalities such as shade, air quality control, and stress relief. The controlled growth in this bio-hybrid system can be viewed as a novel approach of 'Morphogenetic Engineering' [Doursat et al., 2012] that exploits naturally provided mechanisms of growth in plants and extends them with programmable control processes. The developmental approach based on growth processes is common and popular in the artificial life community [Doursat et al., 2012]. Examples are developmental models for artificial neural networks [Jacobi, 1995, Stanley, 2007] and self-assembly [Thenius et al., 2011]. The advantage of our approach is that we have an embodied natural substrate for Morphogenetic Engineering as a starting point (i.e., natural plants) instead of having to design an artificial substrate, such as CPPNs [Stanley, 2007] or aggregating robots [Thenius et al., 2011, Rubenstein et al., 2014]. We can use plants with their known properties of reactions to certain stimuli (tropisms), branching factors, leaf growth patterns, and material properties. Then we go beyond natural growth by imposing artificial stimuli to satisfy requirements concerning shape, material properties, and functions. A constraint of this approach, however, is that we cannot arbitrarily adapt that substrate to our needs but instead have to exploit its given capabilities. The robotic part of the flora robotica system is responsible to push the substrate towards desired shapes and functions that would not be produced by natural growth. The robots and human interactions impose stimuli on the plants that trigger artificial growth of forms. The robots are implemented as hardware modules that allow to implement an artificial growth process and to keep pace with the natural growth of the plants. These robotic assemblies support the biological plants through appropriate scaffolding and also support the plants in maintaining homeostasis. The robot modules are able to control the plants by appropriate stimuli that exploit the plants' different tropisms (e.g., phototropism, hydrotropism, gravitropism). The 


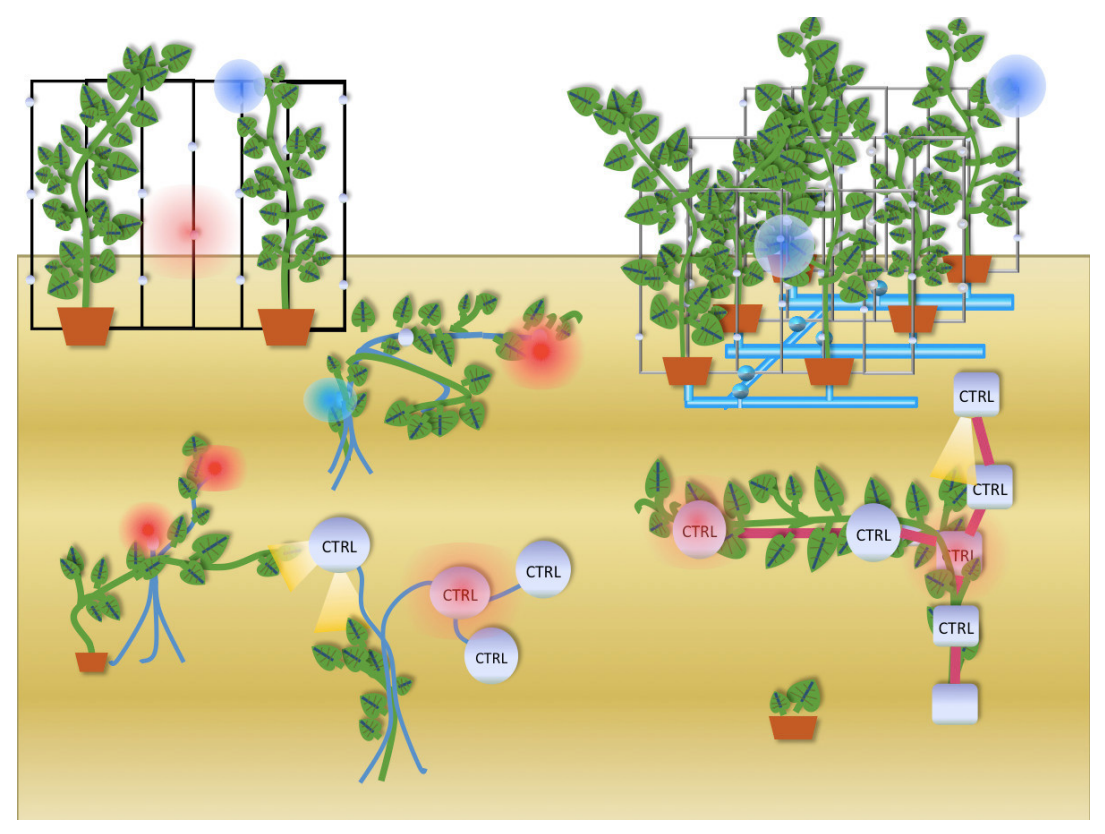

Figure 2: Illustration of a possible instantiation of the envisioned flora robotica system. Robot modules (gray) impose stimuli (red, blue, yellow) on the plants (green). Robots may serve as scaffolding for plants and plants may reinforce these scaffolds.

natural plants, in turn, support and control the robots by guiding them through growth and support their weight in later growth stages. In this way, we explore a developmental plasticity of bio-hybrid systems, where robots and plants grow together from sprout to adult stage while taking different roles during their lifetimes depending on their respective capabilities at their given career stage (see Fig. 2). Hence, they form a closely co-dependent and self-organized system and one can imagine many potential applications of such a setup. In addition to plants and robots in the loop, we also plan to have humans interacting with the bio-hybrid to form, what we call a 'social garden'. Desired structures and behavior patterns emerge based on local interactions. The social garden is our testbed for long-term learning and adaptation where all past actions and interactions between the natural organism and the artificial life systems are represented in the embodiment of the garden (see Sec. 7 for details).

\section{Concept of Controlling plant growth and maintaining homeostasis}

During natural evolution plants as sessile organisms have apdated their growth patterns well to exploit the available physical and nutritional resources, such as light, nutrients, and water [Braam, 2005] with high efficiency. Utilizing modular growth, they show a highest level of developmental and phenotypic plasticity, and are able to modulate growth patterns in response to changes in the environment evoked by physical factors, and by the presence of other organisms [Braam, 2005, Wojtaszek, 2000]. In this project, we explore the modulations of plant growth patterns along and against their evolved capabilities to achieve the intended and adapted shapes and long-term functions. In biology, plants are normally studied under controlled environmental conditions or in the field and in gardening practice, human inter- 


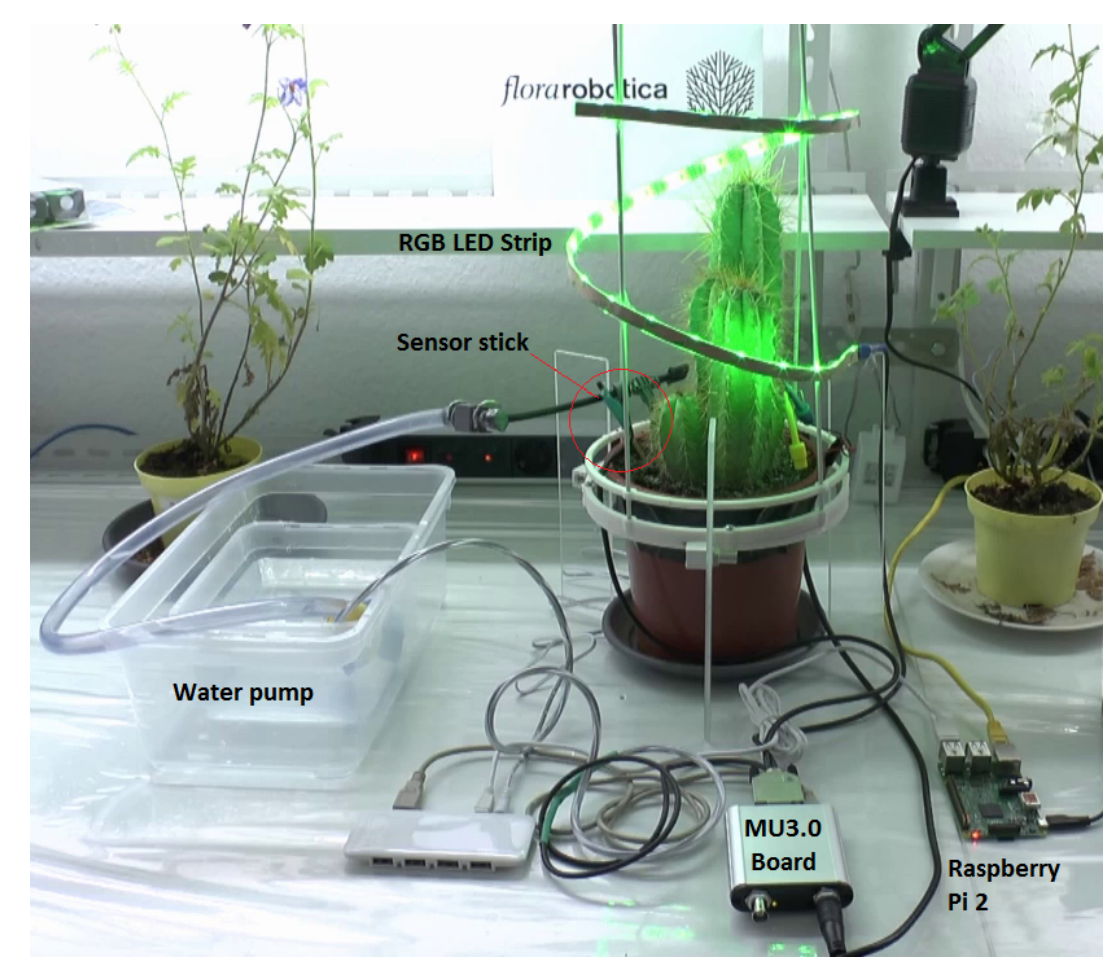

Figure 3: Sensor and actuator setup for maintaining homeostasis; the sensors allow to measure 5 types of data: air temperature, humidity, light intensity, soil moisture, and the electrophysiology of the plant; two types of actuators: water pump and RGB LED strip; pump and pipe system irrigate the soil, the RGB LED strip provides spectral light. The MU3.0 board is a sensor data measurement unit; the Raspberry Pi serves as high-level control unit.

ventions are localized in time and usually synchronized with the phenology of the plants. In our approach, permanent physical and chemical local interactions between robots and plants are expected to result in new emerging properties in the form of global patterns. As means to control and stimulate growth in plants, we make use of the plants' natural response mechanism, in particular, their tropisms. Naturally plants, depending on the respective species, react to light, water, gravity, touch, chemicals, temperature, electrical fields, etc. The key idea of control in this project is to establish a distributed robot system that is controlled in a decentralized organization and operates mainly on local control and locally applied stimuli. Hence, there is the opportunity to create self-organizing control mechanisms that emerge from a close interplay between robots and plants. For example, in later growth stages a robot module can be attached to the plant and is then moved slowly by plant motion and growth. As an effect the stimuli imposed by that robot change in their position and orientation which might feedback to the plant's growth.

In our project we not only manipulate plants but also maintain their homeostasis which is a necessary condition for leveraging the plants' growth. For this purpose we use sensors that perceive the state of plants and their environments (temperature, humidity, environmental light, soil moisture) and actuators that control irrigation, temperature, spectral light and environmental electro-magnetic fields. For example, the use of red LED light is common today to power photosynthesis. 


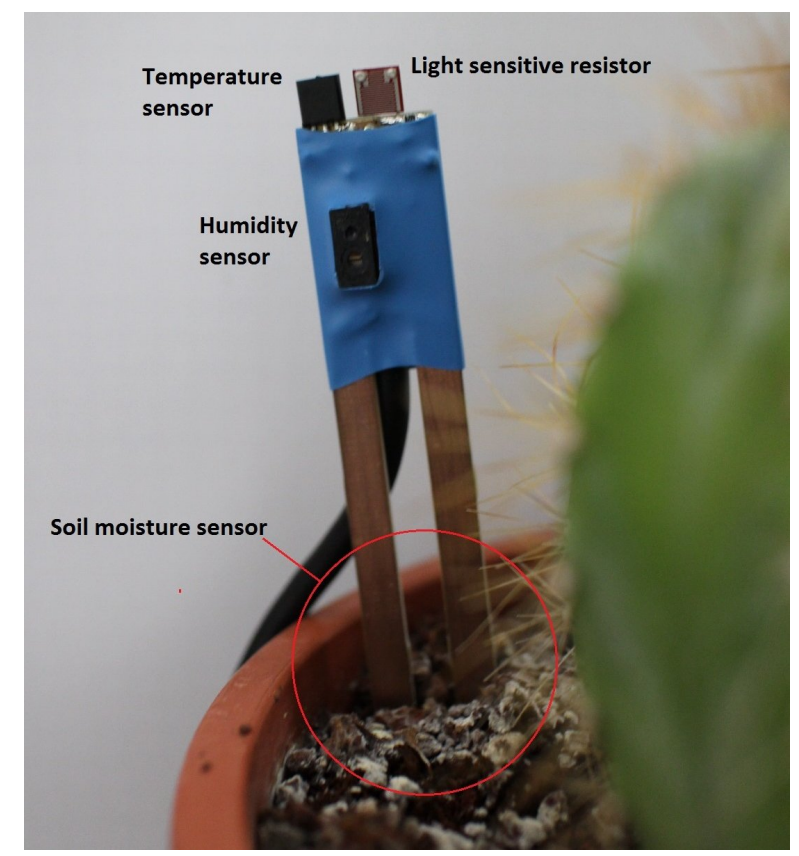

Figure 4: Sensor stick in the plant's soil with temperature sensor, light sensitive resistor, air humidity sensor, and soil moisture sensor.

See Fig. 3 for our prototype setup to maintain the plant's homeostasis. The sensors allow to measure 5 types of data (see Fig. 4): air temperature, humidity, light intensity, soil moisture, and the electrophysiology of the plant. We have two types of actuators: water pump and RGB LED strip. The water pump and a pipe system irrigate the plant's soil, while the RGB LED strip provides spectral light. The sensor data measurement unit called 'MU3.0 board' is our own development. It has high accuracy, low noise (measurement with up to 24 bit ADC resolution), and an actuator controller. The Raspberry $\mathrm{Pi}^{1}$ serves as high-level control of the whole setup and communicates with the MU3.0 board via a virtual COM port. Our approach consists of measuring photosynthetic processes and relating them to the saturation level. Hence, we can investigate the impact of modulated LED light (or modulated electric/magnetic fields) exposed to the plant in ultra-short pulses with different frequencies. As it was shown in experiments, specific frequencies allow selective stimulation of photosynthetic mechanisms of test plants and thus have an impact on growth and development of the plant.

\section{Hardware designs and (phyto-)sensing}

\subsection{Robotic parts}

The robot symbiont of flora robotica takes the form of a scaffold structure assembled from rods and nodes. The rods contain embedded electronics in the form of small autonomous devices. The scaffold is intertwined with the plant symbiont and facilitates interaction between the two. The autonomous devices consist of processing and communications capabilities, an energy harvesting system, sensors, and actuators. The devices are designed to work over

\footnotetext{
${ }^{1}$ https://www.raspberrypi.org/
} 
long periods that match the lifespan of the plant symbiont. Users assemble the scaffold by attaching modules either in response to signals provided by the devices or based on their own needs and goals. Onboard sensors provide information about the plant symbiont, the environment and the status of other devices while actuators allow for interaction with the plant symbiont by exploiting tropisms (light, mechanical stress, chemicals). A picture of a current prototype without the electronics is shown in Fig. 5.

For use during development, debugging and monitoring, a high-powered embedded computer (e.g., BeagleBone Black, Raspberry $\mathrm{Pi}$ ) is placed at the base of the flora robotica system, which is connected to the devices but has different sensors and actuators. It controls biohybrid parts and high power actuators (e.g., LED strips, watering pumps). Additionally, this computer can provide a getaway between an external computer and the swarm simplifying the debugging of the system. We summarize main features of the flora robotica parts:

\subsubsection{Mechanics}

The system contains two different parts: rods and nodes. Rods are the robotic elements with one mechanical connector at both ends. They are comprised of a mechanical structure made of wood or plastic, an electronic board, and a mechanism to harvest energy. Nodes are passive elements where several rods can be attached to form scaffolds. The rods and nodes are connected through a simple press-fit mechanism.

\subsubsection{Electronics and communications}

The electronics are based on an ultra-low power $2.4 \mathrm{GHz}$ wireless System on Chip. It provides low power wireless communications for each rod using protocols based on IEEE standard 802.14.5 which simplifies development and installation. There are no electrical connectors and it allows to update the firmware wirelessly, which is useful for reprogramming and debugging purposes.

\subsubsection{Power}

The rods contain low power circuitry generally in a state of sleep. This allows for the nodes to be powered through harvested energy. Solar energy is harvested using solar panels and techniques such as micro wind turbines or microbial fuel cells. Due to the small size of solar cells, the amount of available energy is limited (especially when placed indoors). The solar cells are initially fitted to one side of the rods but can also be upgraded to form artificial leaves. Besides, each rod has its own energy storage system in order to work autonomously when available energy runs low.

\subsubsection{Sensors}

Regarding proprioceptive sensors, the rods contain a 3D accelerometer and a 3D magnetometer for providing information about the rods orientation and to measure vibrations. Additionally, the rods are able to measure available energy and a flex sensor is used for determining the bending of the rods as an indication of the load supported by the rod. The rod's environmental sensors are comprised of a temperature sensor, buttons to interact with users and capacitive touch sensors for detecting climbing plants attached to the rod. Moreover, the 


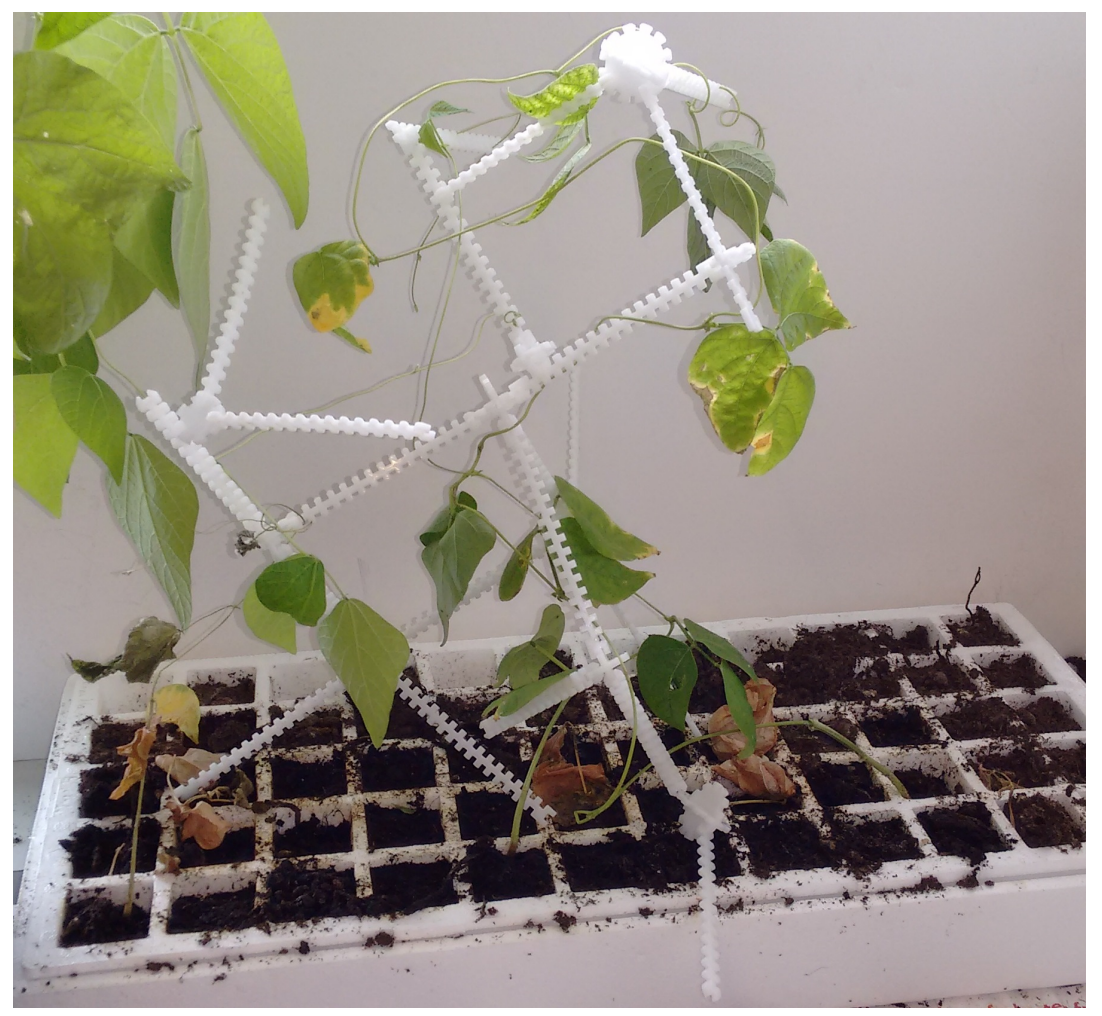

Figure 5: Prototype of the flora robotica rods and nodes without the electronics.

rod has a ring with four integrated ambient light and infrared proximity sensors that detect leafs of the plant symbiont.

\subsubsection{Actuators}

The rods contain only a few actuators due the low power available in the system. They have RGB LEDs and a vibration motor, which is only active for short time intervals. The basic function of the RGB LEDs is to provide feedback to users indicating, for example, when a user should add a new device. In addition, we investigate if LEDs influence the behavior of the plant symbiont. The vibration motor may induce seismonasty in the plant (nastic movements in response to touch stimulus) and may be used to obtain spatial information of the other modules. Releasing hormones in the environment may also yield beneficial applications and are thus considered for implementation in the rods.

\subsection{Bio-hybrid part}

The bio-hybrid part has a set of sensors combined in so-called 'sticks' (e.g., environmental sensors, electrophysiological sensors, photosynthetic sensors, fluidic sensors) and actuators that maintain the plant's homeostasis (watering, chemical additives, spectral light, temperature, EM fields). These sensors and actuators are installed on platforms or on the ground around the plants. We perform a systematic study of effects from those factors on the photosynthetic and growth performances of plants. That enables us to create feedback loops that allow for regulating the imposed stimuli (e.g., extra-lighting), in response to variations of other param- 
eters. Using a specific control methodology, the ad-hoc sensor-actuator phyto-feedback loop allows to maintain an efficient compromise between the needs of plants (i.e., keeping homeostasis) and design purposes of the whole system, such as pushing towards artificial growth processes.

The methodology of using plant sensors and actuators allows for creating specific phytosensors to monitor not only states of plants but also of the environment. In this approach the plant represents a primary, sensitive biological sensor, which perceives different environmental parameters. The secondary technological sensors convert the plant's response into digitally represented information (e.g., by measuring the plant's electrophysiological parameters). This approach, generally denoted as phyto-sensing, represents a powerful way of operating with bio-hybrid systems and at the same time represents a beautiful example of an artificial-life application.

\section{Control methods}

For the control of the bio-hybrid, we develop novel methods for long-term self-adapting and self-repairing growth of robotic structures that influence the plant growth. First, we give a description of the control methods that we plan to apply in this project. Second, we give preliminary results of a simple experiment of controlling the growth and motion of a plant.

\subsection{Methods}

We will extend research on artificial homeostatic hormone systems (AHHS) [Hamann et al., 2012, Schmickl et al., 2011] to create artificial phyto-hormones that might allow for deep insights into growth processes of plants. The controllers utilize a model of physical spreading of substances within the organism and acting based on localized interactions. The artificial substances are influenced by plant signals and act as driving forces for actuators that stimulate and influence plants.

The model of substance flow is extended by a concept of plant growth and combines the control of actuators and the growth model of the mechanical structure. An option is to apply models of plant and animal embryogenic growth that are well studied [Thenius et al., 2011] but rarely used in real-world applications to build physical structures. We expect novel insights into the evolution of growing mechanical structures. Investigations of these novel control paradigms might allow for a deeper understanding of growth processes at a fundamental level. This research track is paralleled by investigations of actual substance flows (e.g., of water, vapor) and distributions of mechanical force with the aim of implementing an embodied control mechanics that leads to coordination/self-regulation.

In contrast to the self-organized control of mobile robots, the growth process of our biohybrids creates permanent structures that constrain possibilities of future growth. Existent control strategies inspired by swarm intelligence are extended to develop new strategies that take long-term effects of current actions into account. The permanent structures of the biohybrid, may provide a positive effect of guidance and forming a long-term embodied memory that is leveraged by the control methods as an embodied world model. The control system of the robotic modules cooperates with the plants following principles of self-organization and it incorporates feedbacks from the plant to achieve long-term learning and adaptation. 


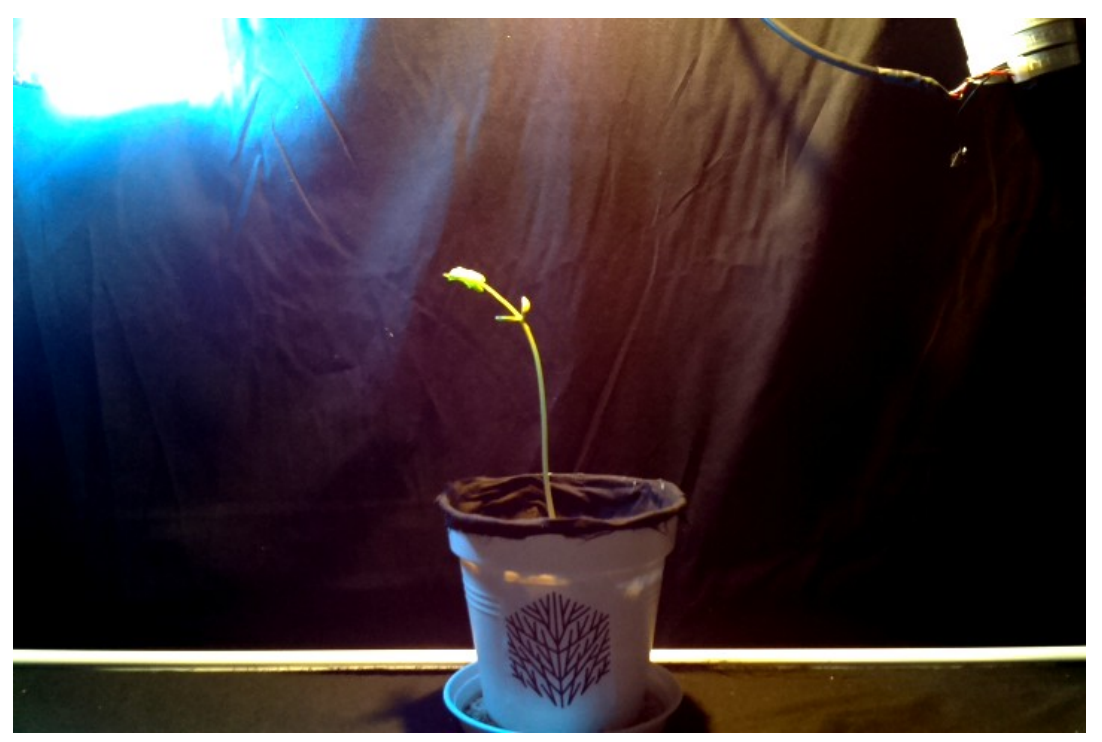

Figure 6: Simple growth and motion experiment: common bean plant (Phaseolus vulgaris), 2 light sources: one at top left (on), one at top right (off).

\subsection{Simple control of plant growth and motion}

We have set up a simple, preliminary experiment of controlling the growth and motion of a plant to demonstrate a few opportunities and constraints of our approach. For our experiments time is always a factor because growth processes are slow. Hence, we require to select a plant that shows fast growth. In addition, we require a plant that also grows well in standard lab conditions or even in office space and is easy to grow. Therefore, we simply use the common bean (Phaseolus vulgaris) in its early growth stage in this preliminary experiment. For our vision of growing architectural artifacts we will consider plants that grow wood later. In the following experiment we have two light sources to impose stimuli on the pant. One light source is located above and left to the plant and the other above and right to the plant. They are turned on in alternating order, each for four hours for a 16 hour period followed by eight hours of darkness. The setup is shown in Fig. 6 .

The light sources are two RGB LED strips with 144 LEDs each. Each LED has a power consumption of $0.2 \mathrm{~W}$ and emits 18 lumen. Each LED strip requires a current of 2.88A. Photos are taken by the Raspberry Pi Camera Module ${ }^{2}$. The LED strips and the camera are controlled by a Raspberry Pi.

In Fig. 7 we show twelve photos of the plant over a period of 38 hours. The experiment is started with a bean plant of about $8 \mathrm{~cm}$ height. In this experiment, the plant had a length of about $22 \mathrm{~cm}$ after 38 hours. Initially the right-hand light is turned on, then the light is switched every four hours. Both are turned off from the 16th to the 24th hour, followed by another 16-hour period of light switching. The photos in Fig. 7 clearly indicate both the growth process of the plant and the plant's motion. During the four-hour period of one light, the plant leans towards it while still maintaining its typical counterclockwise turning behavior (climbing habit, not seen in the photos). In this experiment, the plant's motion towards the left-hand side is more distinct than towards the right-hand side. Also see Fig. 8 for a

\footnotetext{
${ }^{2}$ https://www.raspberrypi.org/products/camera-module/
} 

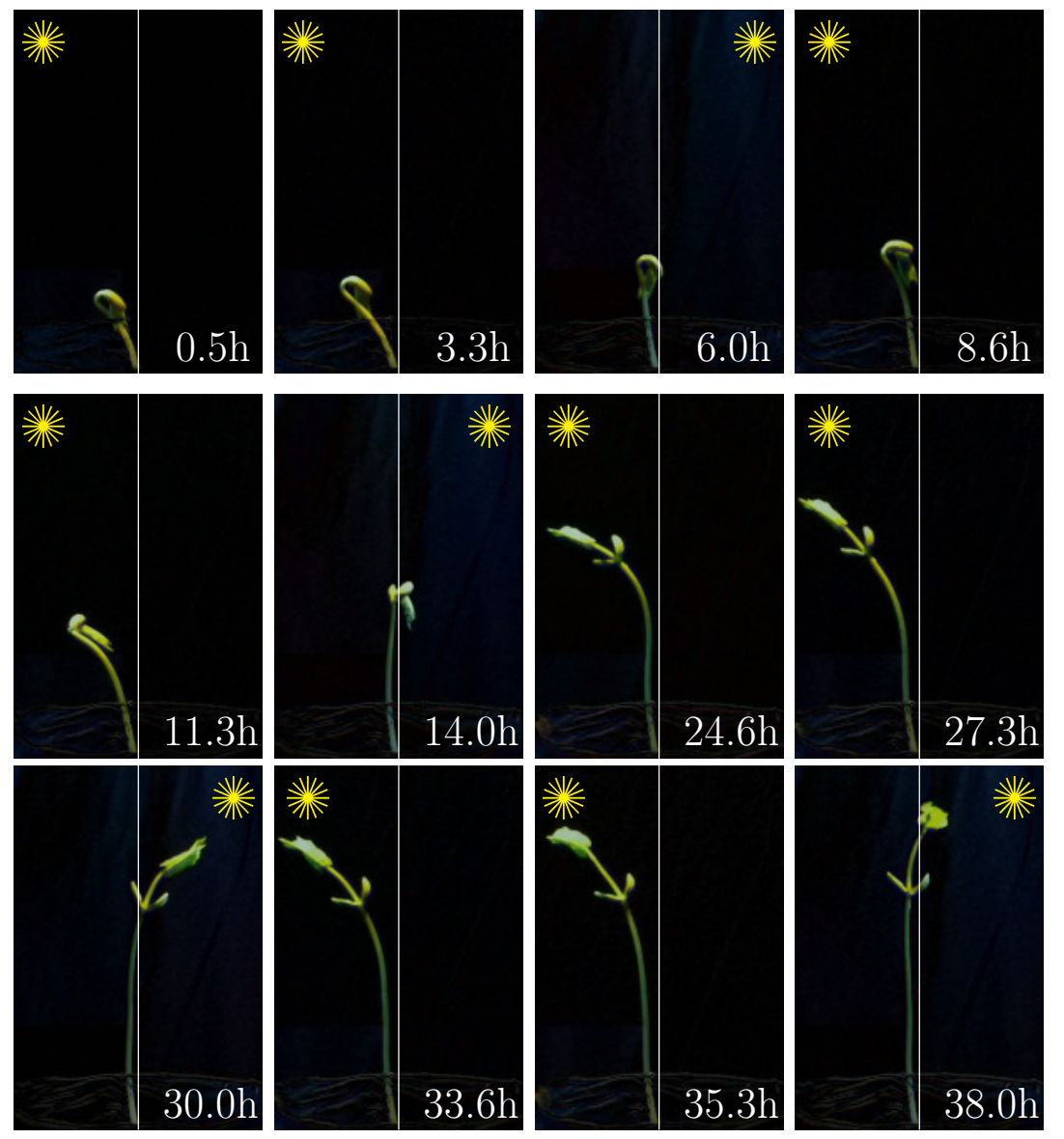

Figure 7: Photos of the bean plant at different times during the experiment showing rapid growth and motion towards light; white line gives position of trunk in soil, sun symbol indicates which light is currently turned on. 


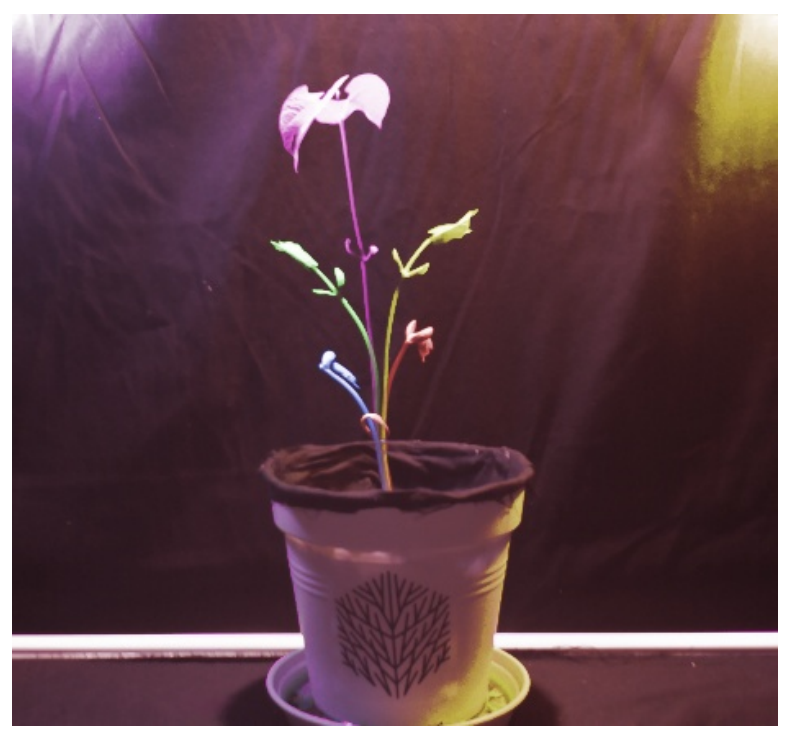

Figure 8: Superposition of photos taken during the experiment in false color indicating the amplitude of the plant's left-right motion and its growth.

superposition of several photos taken during the experiment. It also indicates the amplitude of the plant's left-right motion.

From a series of such experiments we plan to extract main features of the growth process and the effect of controlled stimuli (here: two alternating light sources) on the plant's growth and motion. This gathered data will help us to adapt growth models also common to the artificial life community, such as developmental models similar to L-systems [Lindenmayer, 1975], 'swarm grammars'/interacting growing organisms [von Mammen and Jacob, 2009], and similar approaches [Zamuda and Brest, 2014]. The growth models, in turn, will help us to study our control approaches.

\subsection{Simple control of artificial robot growth}

To test and showcase our general approach, we have performed a simple, preliminary experiment of artificial growth, that is, a growth process of robot structures. As robot platform we temporarily use the Thymio II robot [Riedo et al., 2013]. The robot controller is based on diffusion and production of a simple virtual growth hormone similar to the AHHS approach [Hamann et al., 2012, Schmickl et al., 2011]. Diffusion is implemented by local communication via infra red. The experiment starts with a single robot and additional robots are added manually when requested by a robot within the structure, see Fig. 9. Every robot stores an integer value as the current concentration level of its virtual growth hormone. The growth hormone controls the branching behavior of the growth process. The hormone diffuses from the root and from branching robots to the tips of the branches and forms a gradient along each branch. If the hormone level is lower than a certain threshold in a node, the node produces a certain additional amount of the growth hormone and becomes a branching node. In Fig. 9 four configurations during a growth process are shown with up to 19 Thymio II robots. Initially there is only the root or seed robot which indicates branching by lighting red LEDs (Fig. 9a). Additional robots form two branches (Fig. 9b) and indicate straight 


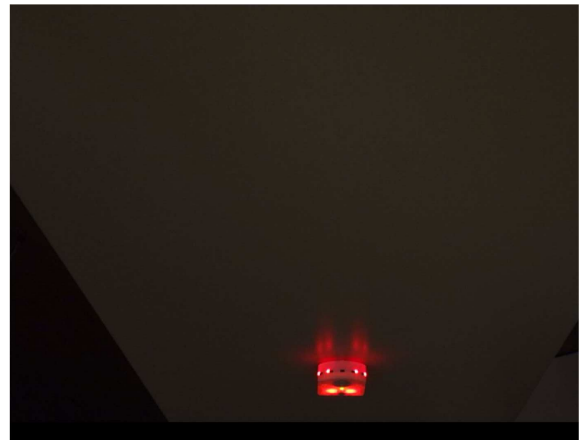

(a)

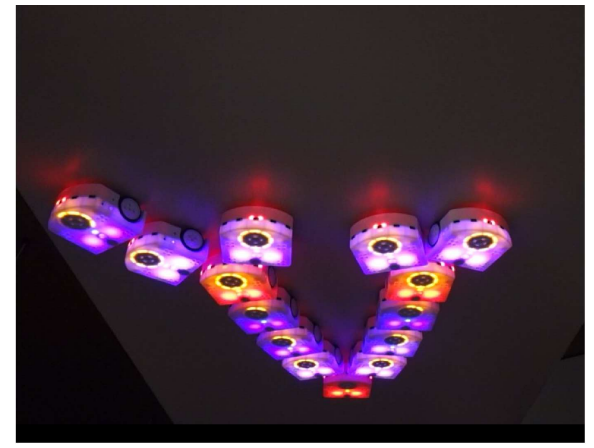

(c)

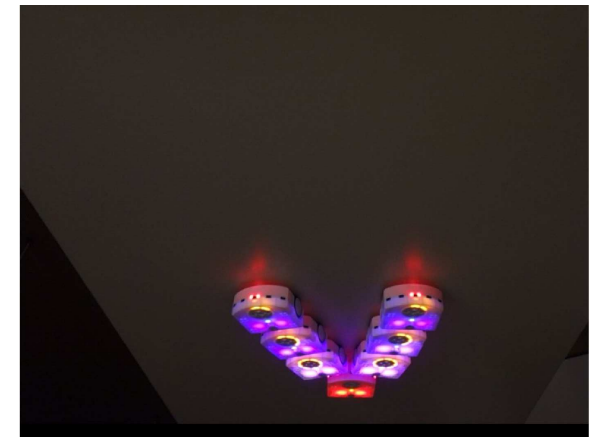

(b)

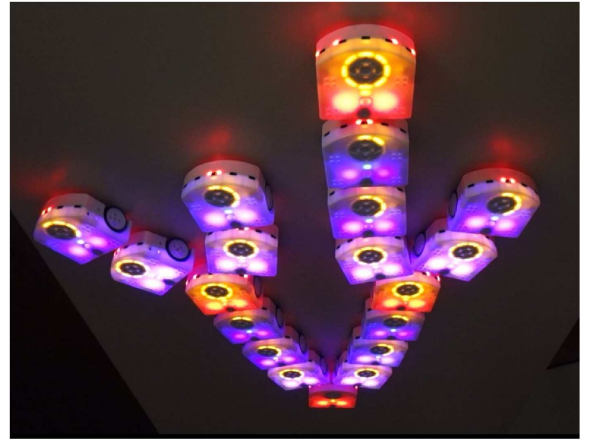

(d)

Figure 9: Preliminary experiment of artificial growth with Thymio II robots (horizontal growth on a table, photos are upside-down), robots indicate branching by red color and stay violet to indicate desired straight growth.

growth by lighting violet LEDs. Then additional branching is again indicated by red LEDs and more robots are added (Fig. 9c). Finally, 19 Thymio II robots have been placed manually in a tree structure with the robot at the top indicating another branching position (Fig. 9d). The system stays dynamic and adaptive. For example, if the root robot of the structure is removed, new seeds emerge and grow new branches.

In this preliminary experiment we test our general approach which is to grow vertical robot structures in synergy with plants. A very early prototype of vertical growth is shown in Fig. 10a and the general concept is shown in Fig. 10b.

\section{Multi-scale representations}

Modeling the flora robotica system requires a special approach because the system relies crucially on different scales, in particular, time scales and scales of behavior. Depending on the plant species the growth process is rather slow compared to the plant's motion or typical velocities of, for example, mobile robots (see Sec. 5.2). It is our vision that future flora robotica systems will grow over dozens of years and undergo different growth stages, what we call a 'growth career'. Effects of plant motion on small time-scales have effects on the growth process on large time-scales and vice versa. An architect might want to know how 


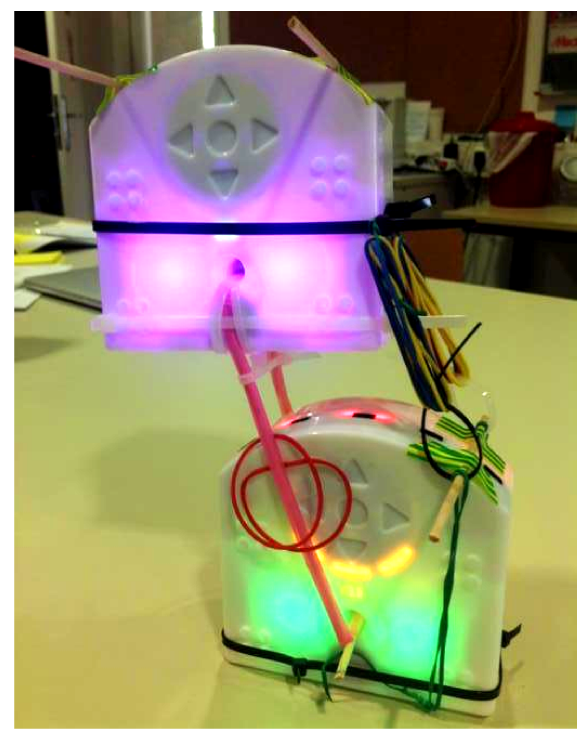

(a) early prototype of artificial vertical growth

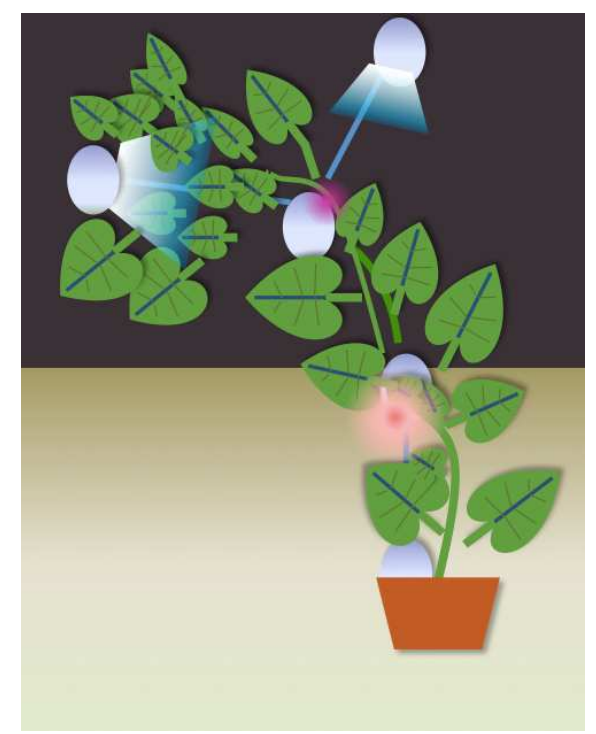

(b) illustration of general bio-hybrid growth concept; plant with robot modules (gray) imposing stimuli (e.g., light)

Figure 10: Early prototype of artificial vertical growth and an illustration of the general concept of bio-hybrid growth.

a flora robotica design will look like in dozens of years, while for control of plant motion a few minutes might make a difference. Similarly the decentralized control approach operates on local control of and by individual robots (microscopic level) and on larger scales of robot clusters (macroscopic level). For example, the overall control of growing biomass might require a macroscopic control approach while adaptations in the direction of growth of a single plant branch might be done locally. As a consequence we also require a multi-scale model approach to test our controllers. At least an approach on the microscopic control level and one on the macroscopic control level should be representable. A scientifically sound approach to designing distributed, self-organizing systems is based on modeling and understanding the micro-macro link, that is, determining the relationship between global and local behavior patterns and vice versa [Schelling, 1978]. The problem of linking the micro- and macro-level applies also to the design of interacting plant-robot systems. Biological models are relevant when minimalist hardware is applied, and there is extensive literature from the field of mathematical biology [Edelstein-Keshet, 2006]. Typical approaches applied in the context of swarm robot systems are that of Martinoli [Martinoli, 1999] and Berman et al. [Berman et al., 2011]. The models developed in this project focus on special features concerning interactions of growing structures. In contrast to models of self-organizing behaviors that focus on mobile agents, here we have moving plant tips interacting with growing robot structures. Our methods address that the past motion of plant tips and robots is embodied in plant and robot structures and hence is persistent. That way an 'embodied memory' emerges that imposes stimuli and constraints on the present and future motion, growth, and behaviors.

Also from an architectural perspective the project breaks new ground and advances knowledge creation in two primary areas related to the concept of multi-scale representations: Practices of Representation and Practices of Construction. 


\subsection{Practices of Representation}

The project challenges prevailing notions of architectural design as focused upon the design and production of 'end-points' - completed spaces, buildings and structures [Burry, 2013]. The challenge is to develop a representational modeling space that supports the design of systems in continual change. The state-of-the-art finds its origins in the relatively recent digitization of architectural design tools and the subsequent maturation of practices from computerization to computation [Terzidis, 2011]. This has resulted in a conceptual shift away from considering architecture as object and towards its consideration as system [Kolarevic, 2003]. While processes such as emergence and self-parameterization are now utilized in the design of architecture, their use still tends towards the design of 'endpoints' - a resultant building, structure or space. This project radically extends the roles and values of architectural representation in three domains: the duration of active influence representations hold in relation to the represented; the means, methods and media by which representations are constructed and used; what it is that is/needs to be represented.

\subsection{Practices of Construction}

The project fundamentally challenges orthodox principles of architectural construction at every level. Building culture is largely predicated on the use of pre-manufactured components and/or pre-processed materials, which are combined by a skilled workforce to make material assemblies in accordance to pre-determined instruction [Groak, 1992]. This project deals with material that is undergoing continual autonomous growth over extended periods. Principles of horticulture become more relevant than principles of building culture. State-of-the-art research in this narrow field - the use of living plants to create load-bearing architectural structures - is characterized by the use of pre-determined and fixed scaffolds and/or the introduction of passive technical components around which plant growth occurs [Ludwig et al., 2012]. This project extends the state-of-the-art by considering reciprocal relations between the vegetal and the technical where both are actively responsive.

\section{Social garden}

In this project we plan to construct an architectural space to promote human/plant symbiont interactions and stimulate development of cultural practices specific to flora robotica. The space is a Social Garden aimed at communicating the sensitive dependencies constructed between environment, plant, robot and social interactions. The Social Garden has physical and digital presence. The physical presence incorporates all sites of physical flora robotica and connects these over the Internet. These spaces permit direct interaction with flora robotica. The digital component of the Social Garden is Internet-based and acts as a searchable data repository from the physical sites. The database is linked to a virtual evolutionary space used to evolve control algorithm parameters. The fitness evaluation of individuals is based on a mix of simulation and real flora robotica data and the fitness value is based on a mixed initiative between fitness functions and user feedback (i.e., subjective fitness). We plan to develop a graphical user-interface to allow users to browse the designs of others. This crowdsourced design process allows collaborative invention of new types of flora robotica. A Social Garden message board supports the sharing of infomration about the virtual plant symbiont and provides a social link between users. The Social Garden also acts as a primary dissemination 


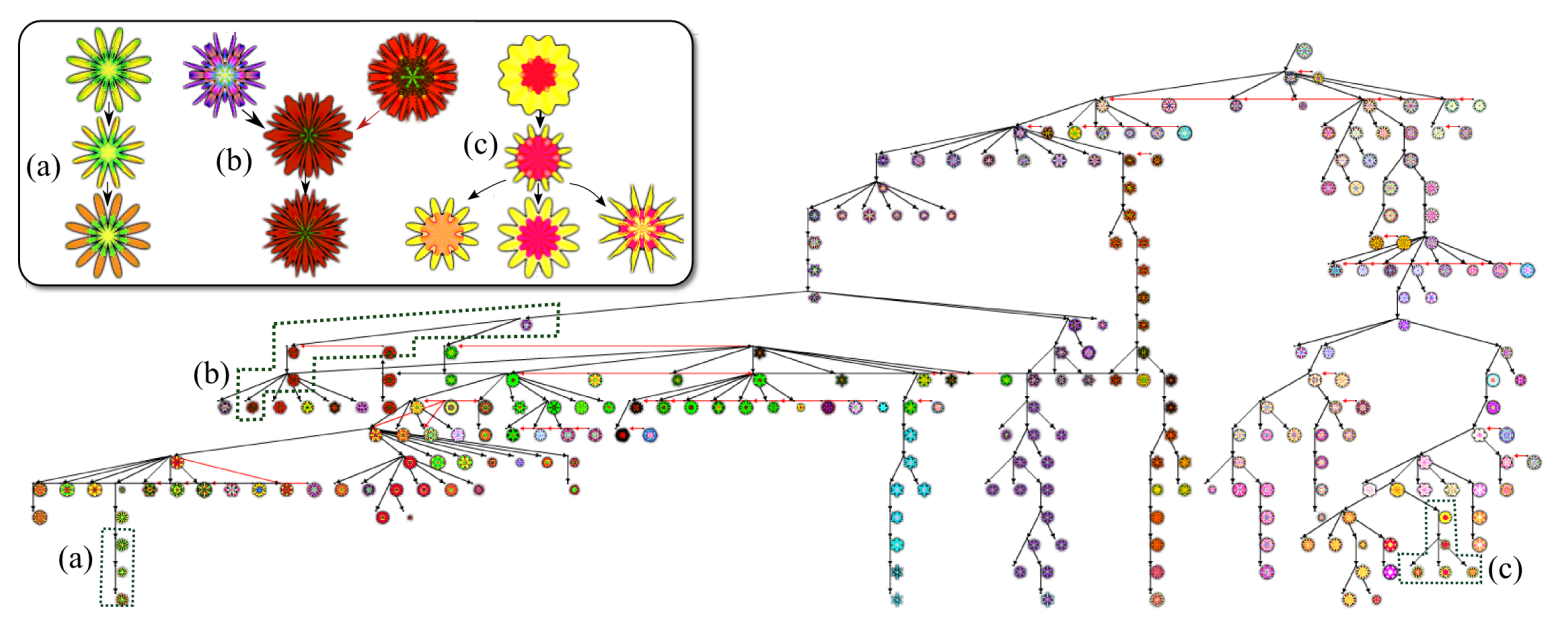

Figure 11: A Phylogeny of Interactively Evolved Flowers. This tree depicts the efforts of 13 different users that evolved flower designs together in a game called Petalz [Risi et al., 2015]. Allowing users to collaborate in a similar manner in flora robotica's Social Garden should enable them to explore a large design space than each user could explore on its own (figure from [Risi et al., 2015]).

space for the project.

While growing house-sized plant-robot hybrids in the real world is out of the timeline of this project, the digital presence of the social garden allows humans to get a glimpse of this vision that they can interact with. We envision a simulated game-like environment running on the web in which users can see their hybrids grow, run their own evolutionary experiments, and collaborate with other users on the design of hybrids. Importantly, such collaborative exploration can lead to a proliferation of different designs (Fig. 11) and can in fact also create new social links between users [Risi et al., 2015]. It is also possible to import existing physical flora robotica hybrids into the simulation and users that own specific plant sensors are able to connect them to the behavior of their virtual plants.

Given recent developments, it is also not unreasonable to expect that advances in augmented reality technology (AR) could soon allow humans to get an even more impressive demonstration of flora robotica. AR could allow humans to plant virtual flora robotica hybrids in their own houses, see them grow, fast forward for tens or hundreds of years and actually experience robot-plant life as it could be.

\section{Conclusion}

In this paper, we have presented the research agenda and motivation of our interdisciplinary project 'flora robotica'. For the Alife research community it is of interest because it is the creation of mixed societies between 'hard' artificial and 'wet' natural life, it is an original real-world implementation of a morphogenetic engineering approach, and in addition it even is a real-world application of Alife methods. The main impact of this project will be the development of a methodology to create and maintain bio-hybrid, self-organizing system specialized to plant growth and distributed robot systems. The relevant time-scales will be 
different from experiments of mixed societies with animals and a particular focus will be on long-term development of the system also in interaction with human beings.

\section{Acknowledgments}

Project 'flora robotica' has received funding from the European Union's Horizon 2020 research and innovation program under the FET grant agreement, no. 640959.

\section{References}

ASSISI $\left.\right|_{b f}$. Animal and robot societies self-organise and integrate by social interaction (bees and fish), project website, 2015. http://assisi-project.eu.

Spring Berman, Vijay Kumar, and Radhika Nagpal. Design of control policies for spatially inhomogeneous robot swarms with application to commercial pollination. In Steven LaValle, Hirohiko Arai, Oliver Brock, Han Ding, Christian Laugier, Allison M. Okamura, Spiridon (Spyros) Reveliotis, Gaurav S. Sukhatme, and Yasushi Yagi, editors, IEEE International Conference on Robotics and Automation (ICRA'11), pages 378-385, Los Alamitos, CA, 2011. IEEE Press.

J. Braam. The life of plants. New Phytologist, 165:373-389, 2005.

J. Burry. Introducing the dynamics of design: Mechanisms and teleology. In J. Burry, editor, Designing the Dynamic, pages 10-15. Melbourne Books, 2013.

Nikolaus Correll, Nikos Arechiga, Adrienne Bolger, Mario Bollini, Benjamin Charrow, Adam Clayton, Felipe Dominguez, Kenneth Donahue, Samuel Dyar, Luke Johnson, et al. Building a distributed robot garden. In Intelligent Robots and Systems, 2009. IROS 2009. IEEE/RSJ International Conference on, pages 1509-1516. IEEE, 2009.

Nikolaus Correll, Nikos Arechiga, Adrienne Bolger, Mario Bollini, Ben Charrow, Adam Clayton, Felipe Dominguez, Kenneth Donahue, Samuel Dyar, Luke Johnson, et al. Indoor robot gardening: design and implementation. Intelligent Service Robotics, 3(4):219-232, 2010.

Rodrigo da Silva Guerra, Hitoshi Aonuma, Koh Hosoda, and Minoru Asada. Behavior change of crickets in a robot-mixed society. Journal of Robotics and Mechatronics, 22(4):526-531, 2010 .

René Doursat, Hiroki Sayama, and Olivier Michel, editors. Morphogenetic Engineering: Toward Programmable Complex Systems. Springer, 2012.

Leah Edelstein-Keshet. Mathematical models of swarming and social aggregation. Robotica, 24(3):315-324, May 2006.

Paco Calvo Garzón and Fred Keijzer. Plants: Adaptive behavior, root-brains, and minimal cognition. Adaptive Behavior, 19(3):155-171, 2011.

A. Gribovskiy, J. Halloy, J.-L. Deneubourg, H. Bleuler, and F. Mondada. Towards mixed societies of chickens and robots. In Intelligent Robots and Systems (IROS), 2010 IEEE/RSJ International Conference on, pages 4722-4728, Oct 2010. doi: 10.1109/IROS.2010.5649542. 
S. Groak. The Idea of Building. E \& FN Spon, 1992.

José Halloy, G. Sempo, G. Caprari, C. Rivault, M. Asadpour, F. Tâche, I. Saïd, V. Durier, S. Canonge, J. M. Amé, C. Detrain, N. Correll, A. Martinoli, Francesco Mondada, R. Siegwart, and J. L. Deneubourg. Social integration of robots into groups of cockroaches to control self-organized choices. Science, 318(5853):1155-1158, November 2007. doi: 10.1126/science.1144259. URL http://dx.doi.org/10.1126/science.1144259.

Heiko Hamann, Thomas Schmickl, and Karl Crailsheim. A hormone-based controller for evaluation-minimal evolution in decentrally controlled systems. Artificial Life, 18(2):165198, 2012. URL http://dx.doi.org/10.1162/artl_a_00058.

N. Jacobi. Harnessing morphogenesis. In S. Kauffman, editor, Proceeding of information processing in cells and tissues, pages 29-41. IEEE Press, 1995.

B. Kolarevic. Architecture in the Digital Age: Design and Manufacturing, Digital Morphogenesis, chapter Digital Morphogenesis. Spon Press, 2003.

LEURRE. Artificial life control in mixed-societies, project website, 2015. http://leurre.ulb.ac.be.

Aristid Lindenmayer. Developmental algorithms for multicellular organisms: A survey of L-systems. Journal of Theoretical Biology, 54(1):3-22, 1975.

F. Ludwig, H. Schwertfeger, and O. Storz. Living systems: Designing growth in Baubotanik. Material Computation, Architectural Design, 82(2):82-87, 2012.

Alcherio Martinoli. Swarm Intelligence in Autonomous Collective Robotics: From Tools to the Analysis and Synthesis of Distributed Control Strategies. PhD thesis, Ecole Polytechnique Fédérale de Lausanne, 1999.

Achim Menges and Steffen Reichert. Material capacity: Embedded responsiveness. Architectural Design, 82(2):52-59, 2012.

PLEASED. Plants employed as sensing devices, project website, 2015. http://pleased-fp7.eu/.

Fanny Riedo, Morgane Chevalier, Stephane Magnenat, and Francesco Mondada. Thymio ii, a robot that grows wiser with children. In IEEE Workshop on Advanced Robotics and its Social Impacts (ARSO 2013), pages 187-193. IEEE, 2013.

S. Risi, J. Lehman, D.B D'Ambrosio, R. Hall, and K.O Stanley. Petalz: Search-based procedural content generation for the casual gamer. IEEE Transactions on Computational Intelligence and AI in Games, PP(99):1-1, 2015. ISSN 1943-068X. doi: 10.1109/TCIAIG.2015.2416206.

Michael Rubenstein, Alejandro Cornejo, and Radhika Nagpal. Programmable self-assembly in a thousand-robot swarm. Science, 345(6198):795-799, 2014. doi: 10.1126/science.1254295. URL http://www. sciencemag.org/content/345/6198/795. abstract.

Thomas C. Schelling. Micromotives and macrobehavior. WW Norton \& Company, 1978. 
Thomas Schmickl, Heiko Hamann, and Karl Crailsheim. Modelling a hormone-inspired controller for individual- and multi-modular robotic systems. Mathematical and Computer Modelling of Dynamical Systems, 17(3):221-242, 2011.

Grgory Sempo, Stphanie Depickre, Jean-Marc Am, Claire Detrain, Jos Halloy, and Jean-Louis Deneubourg. Integration of an autonomous artificial agent in an insect society: Experimental validation. In Stefano Nolfi, Gianluca Baldassarre, Raffaele Calabretta, John C.T. Hallam, Davide Marocco, Jean-Arcady Meyer, Orazio Miglino, and Domenico Parisi, editors, From Animals to Animats 9, volume 4095 of Lecture Notes in Computer Science, pages 703712. Springer Berlin Heidelberg, 2006. ISBN 978-3-540-38608-7. doi: 10.1007/11840541_58. URL http://dx.doi.org/10.1007/11840541_58.

D.C. Slaughter, D.K. Giles, and D. Downey. Autonomous robotic weed control systems: A review. Computers and electronics in agriculture, 61(1):63-78, 2008.

Kenneth O. Stanley. Compositional pattern producing networks: A novel abstraction of development. Genetic Programming and Evolvable Machines, 8(2): 131-162, 2007. ISSN 1389-2576. doi: 10.1007/s10710-007-9028-8. URL http://dx.doi.org/10.1007/s10710-007-9028-8.

K. Terzidis. Algorithmic form. In A. D. Reader, A. Menges, and S. Ahlquist, editors, Computational Design Thinking, pages 94-101. John Wiley \& Sons, 2011.

R. Thenius, M. Dauschan, T. Schmickl, and K. Crailsheim. Re-generative abilities in modular robots using virtual embryogenesis. In Adaptive and Intelligent Systems, pages 227-237. Springer, 2011.

D'Arcy Wentworth Thompson. On Growth and Form. Cambridge University Press, 1917.

Sebastian von Mammen and Christian Jacob. The evolution of swarm grammars - growing trees, crafting art, and bottom-up design. IEEE Computational Intelligence Magazine, 4 (3):10-19, 2009

P. Wojtaszek. Genes and cell walls: a difficult relationship. Biological Reviews, 75:437-475, 2000 .

A. Zamuda and J. Brest. Vectorized procedural models for animated trees reconstruction using differential evolution. Information Sciences, 278:1-21, 2014. doi: 10.1016/j.ins.2014.04.037. 